\title{
Prevalence and acquisition rate of extended spectrum beta lactamase producing gram- negative organisms (ESBL-GNO) in general medical patients in Switzerland
}

\author{
J Pasricha ${ }^{1 *}$, T Koessler $^{2}$, V Camus ${ }^{1}$, S Harbarth ${ }^{3}$, G Renzi ${ }^{4}$, J Schrenzel ${ }^{4}$, D Pittet ${ }^{1}$, A Perrier $^{1}$, A Iten ${ }^{1}$ \\ From International Conference on Prevention \& Infection Control (ICPIC 2011) \\ Geneva, Switzerland. 29 June - 2 July 2011
}

\section{Introduction / objectives}

We aimed to determine the prevalence and acquisition rate of ESBL-GNO amongst patients admitted to general medical units at our hospital.

\section{Methods}

Patients consecutively admitted to 13 medical wards from March-June 2010 were screened for ESBL-GNO via rectal swab within 48hours of admission, and 36 hours of discharge.

\section{Results}

Of 1967 patients, swabs were obtained in 1111(56\%) at admission and $491(25 \%)$ at discharge with $441(22 \%)$ having both. Mean age was 64 years and $58 \%$ were male. $6.8 \%(75 / 1111)$ of patients were positive for an ESBL-GNO at admission of whom $86 \%$, for whom data were available (24/28), had an ESBL-GNO detected in the previous 6 months. $3.7 \%(18 / 487)$ of patients acquired an ESBL-GNO, having positive cultures at discharge but not at admission. On univariate regression, acquisition of an ESBL-GNO was associated with admission from home (OR 0.2 [95\%CI 0.1-0.6], $\mathrm{p}=.005$ ), transfer from another unit (OR 8.3[95\% CI 2-33], $\mathrm{p}=.003$ ) and receipt of a first or second-generation cephalosporin (OR 7.2 [95\% CI 1-37],p=.017). Receipt of a first or second-generation cephalosporin was the only factor independently associated with ESBL-GNO acquisition (OR 7.1[95\% CI 1-40], $\mathrm{p}=.03)$. Age, sex, intensive care, provenance and receipt of other antibiotics were not associated with ESBL carriage or acquisition.

\section{Conclusion}

Carriage and acquisition of ESBL-GNO is a problem amongst medical patients at our hospital. No risk factors for ESBL-GNO carriage were identified.

\section{Disclosure of interest}

None declared.

\section{Author details}

${ }^{1}$ Infection Control, University of Geneva Hospital, Geneva, Switzerland. ${ }^{2}$ Internal Medicine, University of Geneva Hospital, Geneva, Switzerland. ${ }^{3}$ University of Geneva Hospital, Geneva, Switzerland. ${ }^{4}$ Central Laboratory Bacteriology, University of Geneva Hospital, Geneva, Switzerland.

Published: 29 June 2011

\section{doi:10.1186/1753-6561-5-S6-O24}

Cite this article as: Pasricha et al.: Prevalence and acquisition rate of extended spectrum beta lactamase producing gram-negative organisms (ESBL-GNO) in general medical patients in Switzerland. BMC Proceedings 2011 5(Suppl 6):O24 